Pacific Journal of Mathematics

WATTS COHOMOLOGY AND SEPARABILITY 


\title{
WATTS COHOMOLOGY AND SEPARABILITY
}

\author{
ANDREW T. KITCHEN
}

\begin{abstract}
A cohomology theory, $H_{K}^{p} A$, for commutative $K$-algebras, $A$, is discussed for the case where $K$ is a field. This was originally introduced by $\mathrm{C}$. $\mathrm{E}$. Watts in connection with rings of continuous functions. $\mathbf{N}$. Greenleaf computed $H_{K}^{p} A$ in the case where $A$ is an extension field of $K$. In this paper it is shown that, for any $K$-algebra $A$, the separable closure of $K$ in $A$ can be identified with $H_{K}^{O} A$. Furthermore Greenleaf's result is extended to a substantial class of local algebras.
\end{abstract}

1. Let $K$ be a field and $A$ a commutative $K$-algebra with unit element 1. In [4] Watts described a cochain complex $C_{K} A$, based on the additive Amitsur complex $F_{K} A$ [3]. He showed that in the case where $K=R$ and $A=C(X)$, the ring of continuous real valued functions on the compact Hausdorff space $X$, the cohomology of this complex is naturally isomorphic to the real Čech cohomology of $X$. At the other extreme Greenleaf in [2] proved the following result. If $L$ is an arbitrary extension field of $K$ then $C_{K} L$ is naturally isomorphic to $F_{L_{s}} L$, where $L_{s}$ is the separable closure of $K$ in $L$. Thus the homology of $C_{K} L$ is trivial, except in dimension zero where $H^{\circ}\left(C_{K} L\right) \cong L_{s}$.

In this paper we investigate further the part separability plays in this theory. Letting $A_{s}$ be the separable closure of $K$ in $A$ (see $\S 2)$ and writing $H_{K}^{p} A$ for $H^{p}\left(C_{K} A\right)$, we prove the following results.

Theorem 1. If $A$ is an arbitrary K-algebra then $H_{K}^{0} A=A_{s}$.

THEOREM 2. Let $A$ be a (not necessarily Noetherian) local $K$ algebra with unique maximal ideal, $m$. Suppose the image of $A_{s}$, under the canonical map of $A$ onto $A / m$, is separably closed in $A / m$; then $C_{K} A$ is naturally isomorphic to $F_{A_{s}} A$.

From Theorem 2 it follows that, for such an algebra, $H_{K}^{p} A=0$ for $p>0$.

At the end of the paper we mention some interesting classes of local algebras which satisfy the hypothesis of Theorem 2.

2. The complex $F_{K} A$ is the additive Amitsur complex [3, §4] with a dimension shift of $1: F_{K}^{p} A$ is the $p+1$ - fold tensor product of $A$ over $K$, and the coboundary map $d^{p}: F_{K}^{p} A \rightarrow F_{K}^{p+1} A$ is given by $d^{p}\left(f_{0} \otimes \cdots \otimes f_{p}\right)=\sum_{i=0}^{p+1}(-1)^{i} f_{0} \otimes \cdots \otimes f_{i-1} \otimes 1 \otimes f_{i} \otimes \cdots \otimes f_{p}$. 
Proposition 1. The complex $F_{K} A$ has zero homology, except in dimension zero where $H^{0}\left(F_{K} A\right) \cong K$.

Proof. See [3, Lemma 4.1].

Let $\mu_{p}: F_{K}^{p} A \rightarrow A$ by $\mu_{p}\left(f_{0} \otimes \cdots \otimes f_{p}\right)=f_{0} \cdots f_{p}$. The subcomplex $N_{K} A$ is defined as follows

$$
N_{K}^{p} A=\left\{f \in F_{K}^{p} A \mid \exists g \in F_{K}^{p} A \text { with } \mu_{p} g \text { a unit and } f g=0\right\},
$$

this is easily seen to be equivalent to Watts' definition. The Watts cohomology is then the homology of the complex $C_{K} A=F_{K} A / N_{K} A$.

An element $f \in A$ is said to be separable over $K$ if there exists a polynomial $p \in K[X]$, such that $p(f)=0$ and $p^{\prime}(f)$ is a unit in $A$. The separable closure, $A_{s}$, of $K$ in $A$ is the set of elements of $A$ which are separable over $K$, it is a subalgebra of $A$ (see $\S 3$, Corollary to Theorem 1).

3. From the definition of $C_{K} A$ it is clear that we can consider $H_{K}^{0} A$ to be embedded in $A$.

Proposition 2. If $A$ is an arbitrary $K$-algebra then $A_{s} \subset H_{K}^{n} A$.

Proof. If $f \in A_{s}$, let $p=a_{n} X^{n}+\cdots+a_{0} \in K[X]$ be such that. $p(f)=0$ and $p^{\prime}(f)$ is a unit. Define

$$
h_{k-1}=\sum_{i=1}^{k} f^{i-1} \otimes f^{k-i} \in F_{K}^{1} A .
$$

Then $\mu_{1} h_{k-1}=k f^{k-1}$. If $g=a_{n} h_{n-1}+\cdots+a_{1} h_{0}$, then $(1 \otimes f-f \otimes 1) g=0$ and $\mu_{1} g=p^{\prime}(f)$. Thus $d^{1} f \in N_{K}^{1} A$, so $f \in H_{K}^{0} A$.

Lemma. If $R$ is the Jacobson radical of $A$ then $R \cap H_{K}^{0} A=0$.

Proof. If $f \in H_{K}^{0} A$, then there exists $g \in F_{K}^{1} A$ such that $\mu_{1} g$ is a unit and $(1 \otimes f-f \otimes 1) g=0$. Suppose $f$ is also in $R$. It then follows that, for each maximal ideal $m$, the image of $f$ under the natural map $\varphi: A \rightarrow A / m(=L)$ is zero. Thus $(1 \otimes f) g^{\prime}=0$ in $L \otimes_{K} A$, where $g^{\prime}$ is the image of $g$ under the map $\varphi \otimes 1: A \otimes_{K} A \rightarrow L \otimes_{K} A$. Now $g^{\prime}$ can be written $g^{\prime}=\sum \lambda_{i} \otimes g_{i}$, where the elements $\lambda_{i} \in L$ are linearly independent over $K$. It then follows that $f g_{i}=0$ for all $i$. As $\mu_{1} g$ is a unit a simple argument shows that, for some $i, \varphi g_{i} \neq 0$. So, for each maximal ideal $m$, there exists $g_{m}$ such that $g_{m} \notin m$ and $f g_{m}=0$. Therefore Ann $(f)=A$ and $f=0$.

Proof of Theorem 1. If $f \in H_{K}^{n} A$ then there exists $g=\sum_{i=1}^{n} g_{i} \otimes h_{i}$ 
$\in F_{K}^{1} A$ such that $\sum g_{i} \otimes h_{i} f=\sum g_{i} f \otimes h_{i}$ and $\mu_{1} g$ is a unit. In fact

$$
\sum g_{i} \otimes h_{i} f^{k}=\sum g_{i} f^{k} \otimes h_{i}
$$

for $k=0,1,2, \cdots$. We can assume that $g_{1}, \cdots, g_{n}$ are linearly independent over $K$, in which case $h_{i} f^{k}$ is in the $K$-module spanned by $h_{1}, \cdots, h_{n}$. It follows that there exists a polynomial $q_{i} \in K[X]$ such that $h_{i} q_{i}(f)=0$. Hence, because $\mu_{1} g$ is a unit, $q(f)=q_{1}(f) \cdots q_{n}(f)=$ 0 . Thus $f$ is algebraic over $K$.

For each maximal ideal $m$, the image of $f$ under $\varphi: A \rightarrow A / m(=L)$ is in $H_{K}^{0} L$. Hence, by Greenleaf's result [2], there exists an irreducible polynomial $p_{m} \in K[X]$ such that $p_{m}(\varphi f)=0$ and $p_{m}^{\prime}(\varphi f) \neq 0$. Now $\varphi f$ satisfies $q$, so $p_{m}$ divides $q$ and there are, therefore, only a finite number of distinct $p_{m}$. Let $p_{1}, \cdots, p_{r}$ be those distinct polynomials and let $p=p_{1} \cdots p_{r}$. Clearly $p(f) \in R \cap H_{K}^{0} A$ so $p(f)=0$. A simple argument shows that $p^{\prime}(f)$ is a unit. Thus $H_{K}^{0} A \subset A_{s}$.

REMARK. The proof shows that $H_{K}^{0} A$, and thus $A_{s}$, can be described as follows: $f \in H_{K}^{\circ} A$ if and only if there exist distinct irreducible separable polynomials $p_{1}, \cdots, p_{r} \in K[X]$ such that $p_{1}(f) \cdots$ $p_{r}(f)=0$.

Corollary. The separable closure, $A_{s}$, of $K$ in $A$ is a K-algebra. Furthermore if $A$ is a local algebra then $A_{s}$ is a field extension of $K$.

Proof. By Theorem 1 we can identify $A_{s}$ with $H_{K}^{0} A$. The first part of the result can then by proved easily once we observe the identity

$$
1 \otimes f g-f g \otimes 1=(1 \otimes f-f \otimes 1)(1 \otimes g)+(f \otimes 1)(1 \otimes g-g \otimes 1) .
$$

If $A$ is local and $f$ is a nonzero element of $H_{K}^{\circ} A$, then the minimal polynomial of $f$, constructed in the proof of Theorem 1 , is clearly irreducible over $K$. Thus the subalgebra $K[f]$ of $H_{K}^{0} A$ is a field, and so $f^{-1} \in H_{K}^{0} A$. Therefore $H_{K}^{0} A$ is a field.

4. The following proposition is proved in [2].

Proposition 3. If $L$ is a separable (algebraic) extension field of $K$ then $N_{K}^{p} L=\operatorname{ker} \mu_{p}$.

Using an inductive argument based on Proposition 2, we can in fact remove the restriction that $L$ be a field. 
Proposition 4. If the field $L$ is separable over $K$ and $A$ is an $L$-algebra, then the natural map $\theta: F_{K} A \rightarrow F_{L} A$ induces an isomorphism, $C_{K} A \cong C_{L} A$.

Proof. The induced map is certainly a surjection. On the other hand, by Proposition 3, the sequence

$$
0 \longrightarrow N_{K}^{p} L \longrightarrow F_{K}^{p} L \longrightarrow L \longrightarrow 0
$$

is exact. Applying the exact functor $F_{K}^{p} A \otimes_{B}\left(\right.$ ), where $B=F_{K}^{p} L$, we obtain the exact sequence

$$
0 \longrightarrow F_{K}^{p} A \otimes_{B} N_{K}^{p} L \longrightarrow F_{K}^{p} A \longrightarrow F_{K}^{p} A \otimes_{B} L \longrightarrow 0 \text {. }
$$

However in $F_{K}^{p} A \otimes_{B} L$

$$
a_{0} \otimes \cdots \otimes \lambda a_{i} \otimes \cdots \otimes a_{p} \otimes 1=a_{0} \otimes \cdots \otimes a_{i} \otimes \cdots \otimes a_{p} \otimes \lambda .
$$

So the map of $F_{K}^{p} A \otimes_{B} L$ onto $F_{L}^{p} A$, induced by taking $a_{0} \otimes \cdots \otimes a_{p} \otimes \lambda$ to $a_{0} \otimes \cdots \otimes \lambda a_{p}$, is an isomorphism. The composition of this map with $1 \otimes \mu_{p}$ is $\theta_{p}$, and the kernel of $\theta_{p}$ is thus the image of $F_{K}^{p} A \otimes_{B} N_{K}^{p} L$ in $F_{K}^{p} A$. It follows therefore that ker $\theta_{p} \subset N_{K}^{p} A$. Suppose $f \in F_{K}^{p} A$ with $\theta_{p} f \in N_{L}^{p} A$; then there exists $g \in F_{K}^{p} A$ such that $\mu_{p} g$ is a unit and $f g \in \operatorname{ker} \theta_{p}$. So there exists $h \in F_{K}^{p} A$, such that $\mu_{p} h$ is a unit and $f g h=0$. Since $\mu_{p} h g=\left(\mu_{p} h\right)\left(\mu_{p} g\right)$ is a unit, $f \in N_{K}^{p} A$. This completes the proof.

A ring in which every zero divisor is nilpotent we will call a $Z D N$ ring.

Proposition 5. Let $A$ and $A^{\prime}$ be K-algebras which are $Z D N$ rings, and let $N$ be the ideal of nilpotents of $A$. Suppose $K$ is separably closed in the field of quotients of $A / N$, then $A \otimes_{K} A^{\prime}$ is a ZDN ring.

Proof. If $B$ is a subring of $A$ then it is a $Z D N$ ring, with ideal of nilpotents $N \cap B$. The domain $B /(N \cap B)$ embeds in $A / N$, so $K$ is separably closed in the quotient field of $B /(N \cap B)$. We can therefore restrict ourselves to a finitely generated subalgebra of $A$, and so assume that $A$ is Noetherian. Let $L$ be the quotient field of $A / N$, then $(A / N) \otimes_{K} A^{\prime} \subset L \otimes_{K} A^{\prime}$. So by [2, Proposition 3] $(A / N) \otimes_{K} A^{\prime}$ is a $Z D N$ ring and hence $N \otimes_{K} A^{\prime}$ is primary. However (0) is a primary ideal of $A$ with associated prime $N$. Thus it follows, putting $E=A$ and $F=B=A \otimes_{K} A^{\prime}$ in [1, Chapter IV, §2.6, Theorem 2], that the associated primes of $(0)$ in $A \otimes_{K} A^{\prime}$ are also the associated primes of $N \otimes_{K} A^{\prime}$. Hence (0) is a primary in $A \otimes_{K} A^{\prime}$ also, and so $A \otimes_{K} A^{\prime}$ is a $Z D N$ ring.

Note that if $A$ is a local ring ( $A$ has a unique maximal ideal $m$ ) 
and $n$ is a positive integer, then $A / m^{n}$ is a $Z D N$ ring.

Proposition 6. Let $A$ be a Noetherian local K-algebra; then the natural map of $F_{K}^{p} A$ into the projective limit (inverse limit) of the system $\left\{F_{K}^{p}\left(A / m^{n}\right)\right\}_{n}$ is an injection.

Proof. As $A$ is Noetherian, $\bigcap_{n=1}^{\infty} m^{n}=0$, and so $A \rightarrow \operatorname{proj} \lim _{n}\left(A / m^{n}\right)$ is an injection. The proof can be completed by induction on $p$, using the following lemma, the demonstration of which is straightforward.

Lemma. If $\left\{M_{i}, f_{j i}\right\}$ and $\left\{N_{i}, g_{j i}\right\}$ are projective systems of $K$ modules ( $K$ a field) indexed over the same directed set, and if $M$ and $N$ are the projective limits of these systems, then the natural map of $M \otimes_{K} N$ into proj $\lim _{i}\left(M_{i} \otimes_{K} N_{i}\right)$ is an injection.

Proposition 7. Let $A$ be a local K-algebra and let $K$ be separably closed in $A / m$. If $z$ is a zero divisor in $F_{K}^{p} A$ then $\mu_{p} z \in m$, and hence $N_{K}^{p} A=0$.

Proof. Suppose $z$ is a zero divisor in $F_{K}^{p} A$; then there exists $w \neq 0$ such that $z w=0$. Choose a finitely generated subalgebra, $B$, of $A$ such that $w$ and $z$ are in $F_{K}^{p} B$. The ideal $B \cap m$ is prime in $B$. So, localizing $B$ at $B \cap m$, we get a local Noetherian subalgebra $B^{\prime}$ of $A$, such that $B^{\prime} \cap m$ is the maximal ideal of $B^{\prime}$, and $z$ and $w$ are elements of $F_{K}^{p}\left(B^{\prime}\right)$. We can therefore assume that $A$ is Noetherian. By Proposition 6, there exists $n$ such that the image of $w$ in $F_{K}^{p}\left(A / m^{n}\right)$ is nonzero. Thus $z^{\prime}$, the image of $z$, is a zero divisor in $F_{K}^{p}\left(A / m^{n}\right)$. However $K$ is separably closed in $A / m$ and so, by induction from Proposition 5, we see that $F_{K}^{\rho}\left(A / m^{n}\right)$ is a $Z D N$ ring. The image $z^{\prime}$ is thus nilpotent and the same is true of $\mu_{p} z^{\prime} \in A / m^{n}$. As the image of $\mu_{p} z$ in $A / m^{n}$ is $\mu^{p} z^{\prime}$, it follows that $\mu_{p} z \in m$.

Proof of Theorem 2. As $A_{s}$ is a field we can apply Proposition 4 to get $C_{K} A \cong C_{A_{s}} A$. However Proposition 7 shows that $C_{A_{s}} A=$ $F_{A_{s}} A$. This completes the proof.

The following corollary to Theorem 2 is immediate on applying Proposition 1.

CoRollary. If A satisfies the hypotheses of Theorem 2, then $H_{K}^{p} A=0$ for $p>0$.

Clearly any local algebra over a separably closed field (i.e. separably closed in its algebraic closure) satisfies the hypotheses of Theorem 2. 
If $A$ is a complete Noetherian local $K$-algebra, there exists [5, Chapter VIII, §12, Theorem 27] a subfield $L$ of $A$ which is mapped onto $A / m$ by the natural map. Under these circumstances $A_{s}$ is mapped isomorphically onto the separable closure of $K$ in $A / m$. Thus it follows that, for such an algebra also, the hypotheses of Theorem 2 are satisfied.

Our ultimate goal is to prove the conclusion of Theorem 2 for all local $K$-algebras; then, loosely speaking, to study this cohomology theory for an arbitrary $K$-algebra by using sheaf theoretic methods to patch the algebra together from its localizations (at prime or maximal ideals). Partial results in this direction have been obtained.

\section{REFERENCES}

1. N. Bourbaki, Algèbra Commutative, Chapter IV, Hermann, Paris, 1961.

2. N. Greenleaf, Watts cohomology of field extensions, Proc. Amer. Math. Soc., 21 (1969), 208-210.

3. A. Rosenberg and D. Zelinski, On Amitsur's complex, Trans. Amer. Math. Soc., 97 (1960), 327-356.

4. C. Watts, Alexander-Spanier cohomology and rings of continuous functions, Proc. Nat. Acad. Sci. U.S.A., 54 (1965), 1027-1028.

5. O. Zariski and P. Samuel, Commutative Algebra, Vol. II, D. Van Nostrand, Princeton, N.J., 1960.

Received May 21, 1971 and in revised form November 23, 1971.

INSTITUTO DE MATEMÁtica

UNIVERSIDADE FEDERAL DO RIO DE JANEIRO

Caixa Postal 1835 ZC-00

20.000 RIO DE JANeIRO, GB 


\section{PACIFIC JOURNAL OF MATHEMATICS}

\section{EDITORS}

H. SAMELSON

Stanford University

Stanford, California 94305

C. R. HobBY

University of Washington

Seattle, Washington 98105
J. DUGUNDJI

Department of Mathematics

University of Southern California

Los Angeles, California 90007

RICHARD ARENS

University of California

Los Angeles, California 90024

\section{ASSOCIATE EDITORS}

E. F. BECKENBACH

B. H. NeumanN

F WoLF

K. YoshidA

\section{SUPPORTING INSTITUTIONS}

UNIVERSITY OF BRITISH COLUMBIA

UNIVERSITY OF SOUTHERN CALIFORNIA

CALIFORNIA INSTITUTE OF TECHNOLOGY

UNIVERSITY OF CALIFORNIA

MONTANA STATE UNIVERSITY

STANFORD UNIVERSITY

UNIVERSITY OF NEVADA

NEW MEXICO STATE UNIVERSITY

OREGON STATE UNIVERSITY

UNIVERSITY OF OREGON

OSAKA UNIVERSITY

UNIVERSITY OF TOKYO

UNIVERSITY OF UTAH

WASHINGTON STATE UNIVERSITY

UNIVERSITY OF WASHINGTON

$\stackrel{*}{*} \stackrel{*}{*} \stackrel{*}{*}{ }^{*}{ }^{*}$ MMEICAN MATHEMATICAL SOCIETY

NAVAL WEAPONS CENTER

Printed in Japan by International Academic Printing Co., Ltd., Tokyo, Japan 


\section{Pacific Journal of Mathematics}

\section{Vol. 42, No. $1 \quad$ January, 1972}

Tage Bai Andersen, On Banach space valued extensions from split faces ........

David Marion Arnold, A duality for quotient divisible abelian groups of finite

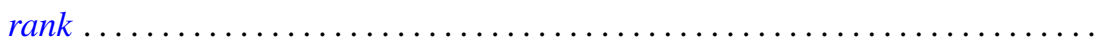

Donald Pollard Ballou, Shock sets for first order nonlinear hyperbolic

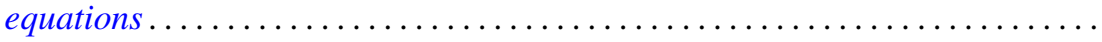

Leon Brown and Lowell J. Hansen, On the range sets of $H^{p}$ functions .........

Alexander Munro Davie and Arne Stray, Interpolation sets for analytic

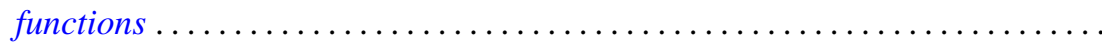

M. G. Deshpande, Structure of right subdirectly irreducible rings. II . . . . . . . . .

Barry J. Gardner, Some closure properties for torsion classes of abelian

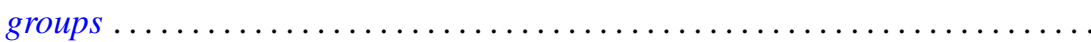

Paul Daniel Hill, Primary groups whose subgroups of smaller cardinality are

direct sums of cyclic groups . . . . . . . . . . . . . . . . . . .

Richard Allan Holzsager, When certain natural maps are equivalences .........

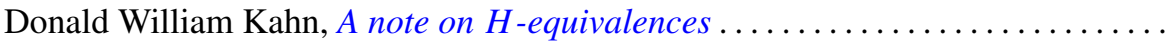

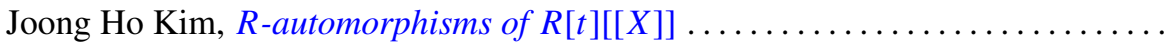

Shin'ichi Kinoshita, On elementary ideals of polyhedra in the 3-sphere.........

Andrew T. Kitchen, Watts cohomology and separability...

Vadim Komkov, A technique for the detection of oscillation of second order

ordinary differential equations .

Charles Philip Lanski and Susan Montgomery, Lie structure of prime rings of characteristic 2

Andrew Lenard, Some remarks on large Toeplitz determinants . .

Kathleen B. Levitz, A characterization of general Z.P.I.-rings. II .

Donald A. Lutz, On the reduction of rank of linear differential systems

David G. Mead, Determinantal ideals, identities, and the Wronskian ...

Arunava Mukherjea, A remark on Tonelli's theorem on integration in product

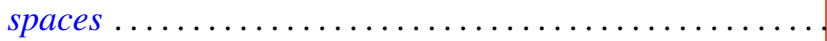

Hyo Chul Myung, A generalization of the prime radical in nonassociative rings.

John Piepenbrink, Rellich densities and an application to unconditionally nonoscillatory elliptic equations.

Michael J. Powers, Lefschetz fixed point theorems for a new class of multi-valued maps .

Aribindi Satyanarayan Rao, On the absolute matrix summability of a Fourier

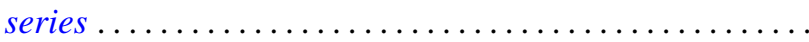

T. S. Ravisankar, On Malcev algebras ......................... 227

William Henry Ruckle, Topologies on sequences spaces . . . . . . . . . . . . . 235

Robert C. Shock, Polynomial rings over finite dimensional rings . . . . . . . . . 251

Richard Tangeman, Strong heredity in radical classes . . . . . . . . . . . . . . 259

B. R. Wenner, Finite-dimensional properties of infinite-dimensional spaces . . . . 267 